\title{
Água tônica: aceitação e análise tempo-intensidade do gosto amargo
}

\author{
Tonic water: acceptance and analysis time-intensity of the bitter taste
Valéria Paula Rodrigues MINIM ${ }^{1 \star}$, Milene Moreira RIBEIRO ${ }^{1}$, Márcia Cristina Teixeira Ribeiro VIDIGAL ${ }^{1}$, Manoela Maciel dos SANTOS ${ }^{1}$, Marília Magalhães GONÇALVES ${ }^{1}$

\section{Resumo}

A água tônica é um refrigerante caracterizado pelo gosto amargo atribuído à presença de quinina. O presente trabalho teve como objetivo avaliar a aceitação e a percepção do gosto amargo de três marcas comerciais de água tônica. Para o teste de aceitação foi utilizada escala hedônica de nove pontos com noventa e seis consumidores. Este estímulo também foi analisado utilizando-se a técnica tempo-intensidade. Nove provadores selecionados e treinados avaliaram as amostras da água tônica utilizando o programa "Sistema de Coleta de Dados TempoIntensidade-SCDTI" para Windows. Os resultados obtidos foram analisados por análise de variância (ANOVA) e teste de Duncan para comparação de médias. As marcas de água tônica apresentaram curvas de tempo-intensidade distintas para o gosto amargo. As amostras não apresentaram diferença quanto à intensidade do gosto amargo pela análise tempo-intensidade. Em relação à aceitação, as amostras M-1 e M-2 diferiram da amostra M-3 ( $\mathrm{p}<0,05)$, sendo M-3 a menos aceita.

Palavras-chave: água tônica; análise tempo-intensidade; aceitação; comparação múltipla.

\begin{abstract}
Tonic water is a soda characterized by a bitter taste attributed to quinine. The objective of this work was to evaluate the acceptance and the perception of the bitter taste of three commercial brands of tonic water. For the acceptance test, a hedonic scale of nine points and ninety six consumers were used. This attribute was also analyzed using the Time-intensity technique. Nine individuals, selected as panelists, evaluated the samples of tonic water using the program "System of Collection of Data Time-intensity-SCDTI " for Windows. The obtained results were analyzed by the Analysis of Variance (ANOVA) and Duncan's test. The brands of tonic water showed differences in Time-intensity curves for the bitter taste. The samples showed no difference regarding the intensity of the bitter taste in the Time-intensity's analysis. The M-1 and M-2 samples were better graded than M-3.

Keywords: tonic water; analysis time-intensity; acceptance; multiple comparison.
\end{abstract}

\section{Introdução}

Água tônica é um refrigerante que contém cerca de 10\% de açúcar e até $85 \mathrm{mg} . \mathrm{L}^{-1}$ de quinina. $\mathrm{O}$ açúcar além da função de adoçar propicia corpo à bebida. Inicialmente, a quinina era adicionada como sendo um composto com efeitos benéficos contra a malária. Atualmente é utilizada apenas para conferir o gosto amargo, característica sensorial marcante deste produto. A água tônica é muito usada como uma bebida de mistura para coquetéis, especialmente os que são feitos com gim (por exemplo, gim-tônica) (WIKIPEDIA, 2007).

A análise tempo-intensidade é um complemento da análise sensorial clássica, já que fornece informações sobre a sensação percebida ao longo do tempo (CLIFF; HEYMANN, 1993). Consiste na medida da velocidade, duração e intensidade percebidas por um único estímulo, através da associação da percepção humana com recursos da informática (REIS, 2007). Esta técnica é importante na avaliação sensorial de um alimento, uma vez que a percepção do aroma, do sabor e da textura é um fenômeno dinâmico e não estático (MONTEIRO, 2002).
Durante a avaliação temporal de um produto, os estímulos sensoriais são caracterizados pelo aumento da percepção seguido por uma intensidade máxima e culminando na sua extinção (KELLING; HALPERN, 1983).

A análise tempo-intensidade tem sido usada como ferramenta para pesquisa com gosto amargo (CARDELLO; DA SILVA; DAMÁSIO, 1999; MONTEIRO, 2002; SILVA et al., 2004). Este estímulo apresenta característica residual, o que torna interessante o estudo da sua percepção temporal. Silva et al. (2004) avaliaram o gosto amargo por meio desta técnica em amostras de café orgânico e convencional.

O gosto amargo na água tônica é fundamental na aceitação deste produto. Desta forma, o objetivo deste trabalho foi avaliar a percepção do gosto amargo de água tônica de diferentes marcas comerciais ao longo do tempo através da técnica tempointensidade e sua relação com a aceitação. 


\section{Material e métodos}

\subsection{Amostras}

Foram avaliadas três marcas comerciais de água tônica (M1, M2, M3) adquiridas em supermercados da cidade de Viçosa, MG.

As avaliações foram realizadas no Laboratório de Análise Sensorial do Departamento de Tecnologia de Alimentos da Universidade Federal de Viçosa. As amostras foram servidas em copos descartáveis, codificados com números de três dígitos, contendo aproximadamente $30 \mathrm{~mL}$ de água tônica à temperatura de $10^{\circ} \mathrm{C}$.

\subsection{Análise tempo-intensidade do gosto amargo}

O programa utilizado para a Análise Tempo-Intensidade foi desenvolvido no Laboratório de Análise Sensorial da Faculdade de Engenharia de Alimentos - UNICAMP denominado Sistema de Coleta de Dados Tempo-Intensidade-SCDTI versão 1.0. O SCDTI opera em ambiente Windows e consiste em um programa para coleta e manipulação de dados tempo-intensidade, no qual o usuário indica utilizando o mouse a atual intensidade do estímulo a ser coletado. Possibilita a escolha do comprimento da escala a ser utilizada, em cada teste, e armazena a sequência de dados lidos para uso futuro. Ainda, permite a manipulação dos dados armazenados, para a obtenção de informações como gráficos, parâmetros das curvas tempo-intensidade como área sob a curva obtida, intensidade máxima do estímulo percebido e tempo total de duração do estímulo. No decorrer da análise, são apresentadas mensagens de aviso do início de novas etapas, com instruções ao provador sobre a ação a ser realizada (CARDELLO; DA SILVA; DAMASIO, 1999).

O tempo estipulado para a realização da análise de tempointensidade foi de 90 segundos, incluindo 10 segundos em que provador permanecia com a amostra na boca antes de engoli-la. Este tempo foi estipulado após a avaliação das três amostras pelos provadores. O tempo de permanência do estímulo gosto amargo foi cronometrado para cada amostra em três repetições. A média do tempo de maior duração do estímulo foi acrescida de trinta segundos e utilizada como tempo total a fim de garantir que a permanência do atributo estivesse dentro do período de análise.

\section{Recrutamento e pré-seleção de provadores}

Os provadores foram recrutados por meio de um questionário sendo selecionados aqueles que possuíam interesse em realizar os testes, disponibilidade de tempo para as análises, habilidade com o computador e uso de escalas, que não apresentavam problemas de saúde que afetassem a análise e que gostam do produto.

A pré-seleção dos provadores foi realizada por meio do teste triangular em quatro repetições em que vinte e um julgadores avaliaram duas soluções contendo cafeína nas concentrações fraco $(0,04 \%)$ e forte $(0,20 \%)$. As amostras foram casualizadas e balanceadas segundo delineamento experimental apropriado.
O critério de seleção consistiu na obtenção de pelo menos 75\% de respostas corretas.

\section{Determinação do atributo e treinamento dos provadores}

O gosto amargo foi definido como o atributo de maior relevância nas amostras de água tônica.

Os provadores passaram por algumas sessões de treinamento para memorização da sensação percebida do gosto amargo forte e familiarização com o programa SCDTI, a fim de que pudessem aprimorar a coordenação motora e utilizar o mouse em sincronia com a percepção do atributo. Todos os procedimentos em relação ao programa SCDTI foram padronizados nesta etapa de modo que os provadores não fossem influenciados.

Foi utilizado como padrão de referência para gosto amargo nenhum a água mineral gasosa e para gosto amargo forte a Água Tônica Schweeps.

\section{Seleção final dos provadores}

A seleção da equipe final de provadores foi realizada por meio de um teste preliminar com as três amostras de água tônica. As amostras foram avaliadas em três repetições, sendo apresentadas de forma monádica. Os resultados obtidos por provador e para cada parâmetro da curva tempo-intensidade foram analisados pela análise de variância (ANOVA). Foram dispensados os provadores que obtiveram probabilidade para $\mathrm{F}_{\text {repeticão }}$ menor ou igual a 0,05 em pelo menos um dos parâmetros (MONTEIRO, 2002).

A probabilidade de $\mathrm{F}_{\text {amostra }}$ não consistiu em um critério para eliminação dos provadores, uma vez que após realização de um teste de comparação múltipla não foi observada diferença significativa entre as três marcas de água tônica em relação à intensidade do gosto amargo $(\mathrm{p}>0,05)$.

\section{Avaliação das amostras e parâmetros da curva tempo-intensidade}

As amostras foram apresentadas, de forma monádica e aleatória, aos nove provadores selecionados, em três repetições. Todas as instruções foram fornecidas pelo programa SCDTI, para que o provador colocasse a amostra na boca e engolisse após os 10 segundos previamente estipulados. A escala utilizada para a análise foi de nove pontos, sendo $0=$ nenhum gosto amargo e 9 = gosto amargo forte.

Os parâmetros avaliados da curva foram: TImax (tempo de intensidade máxima), Imax (intensidade máxima), Platô (tempo de duração da intensidade máxima), Área (área sob a curva), Td (tempo correspondente ao ponto em que a intensidade máxima começa a declinar) e Ttot (tempo total de duração do estímulo).

\section{Análise dos resultados}

Foi realizada uma análise de variância (ANOVA) para os dados obtidos dos parâmetros avaliados, utilizando-se amostra e provador como fontes de variação e interação para 
cada parâmetro e teste de Duncan para comparação de médias $(\alpha=0,05)$. As análises estatísticas foram realizadas utilizando-se o programa estatístico SAS (Statistical Analysis System, 1999), versão 9.1, licenciado para a Universidade Federal de Viçosa.

\subsection{Teste de aceitação}

Foi realizado um teste de aceitação das três marcas de água tônica utilizando escala hedônica de nove pontos (MINIM, 2006). As amostras devidamente codificadas aleatoriamente com números de três dígitos foram servidas de forma aleatória aos noventa e seis provadores em cabines individuais em uma única sessão. Junto com as amostras foi servido um copo de água filtrada em temperatura ambiente para que os provadores pudessem enxaguar a boca entre as avaliações.

A análise dos resultados foi feita através da análise de variância (ANOVA) e teste de Duncan para comparação de médias $(\alpha=0,05)$ utilizando-se o programa estatístico SAS (Statistical Analysis System) (SAS, 1999), versão 9.1, licenciado para a Universidade Federal de Viçosa.

\section{Resultados e discussão}

\subsection{Análise tempo-intensidade}

Entre os vinte e um provadores recrutados por meio dos questionários, dez obtiveram $75 \%$ de acerto no teste triangular e foram pré-selecionados, sendo cinco homens e cinco mulheres na faixa etária de 20 a 40 anos.

Após o treinamento, os dez provadores pré-selecionados foram avaliados por meio do teste preliminar com as três amostras de água tônica. As probabilidades de $\mathrm{F}_{\text {repetição }}$ que determinam a repetibilidade dos resultados dos provadores para os parâmetros da curva, são apresentadas na Tabela 1.

O provador 6 foi dispensado, pois apresentou probabilidade de $\mathrm{F}_{\text {repetição }}$ inferior a 0,05 no parâmetro intensidade máxima. Os outros nove provadores (quatro mulheres e cinco homens) foram selecionados para a avaliação final das amostras de água tônica para o atributo gosto amargo.

Tabela 1. Níveis de probabilidade de $\mathrm{F}_{\text {repeticăo }}$ dos provadores para os parâmetros da curva tempo-intensidade.

\begin{tabular}{ccccccc}
\hline Provador & Timax & Imax & Área & Td & Platô & Ttot \\
\hline 1 & 0,30 & 0,27 & 0,93 & 0,30 & 0,25 & 0,45 \\
2 & 0,84 & 0,65 & 0,57 & 0,86 & 0,89 & 0,54 \\
3 & 0,64 & 0,90 & 0,21 & 0,77 & 0,46 & 0,25 \\
4 & 0,80 & 0,54 & 0,45 & 0,25 & 0,15 & 0,54 \\
5 & 0,87 & 0,40 & 0,23 & 0,68 & 0,78 & 0,24 \\
6 & 0,85 & 0,00 & 0,90 & 0,90 & 0,80 & 0,90 \\
7 & 0,47 & 0,94 & 0,12 & 0,44 & 0,70 & 0,66 \\
8 & 0,24 & 0,56 & 0,80 & 0,88 & 0,92 & 0,79 \\
9 & 0,56 & 0,63 & 0,76 & 0,64 & 0,85 & 0,55 \\
10 & 0,15 & 0,87 & 0,68 & 0,08 & 0,44 & 0,06 \\
\hline
\end{tabular}

Probabilidade menor ou igual a 0,05 indica que a repetibilidade do provador não está satisfatória para o critério de corte estabelecido.
Na Tabela 2 encontra-se o resultado do teste de comparação de médias (Duncan) dos parâmetros da curva tempointensidade.

As amostras de água tônica não diferiram entre si para os parâmetros Imax, Ttot, Área e TImax ao nível de 5\% de significância. Isto indica que as amostras apresentam a mesma intensidade máxima (Imax) para o atributo avaliado. Além disto, essa intensidade é alcançada no mesmo tempo (TImax) para as três amostras avaliadas. O tempo total de permanência do gosto amargo (Ttot) e a relação da intensidade com o tempo de permanência (Área), relação que fornece a área sob a curva, foram estatisticamente iguais para as amostras em estudo. A amostra M-1 diferiu das demais em relação ao parâmetro platô, apresentando menor valor, ou seja, a amostra M-1 permanece menos tempo na intensidade máxima do atributo gosto amargo. Já em relação ao Td, ou seja, o tempo no qual a intensidade do gosto amargo começa a diminuir, a amostra $\mathrm{M}-1$ diferiu da $\mathrm{M}-2$, e estas não diferiram da amostra M-3. Este resultado confirma o teste de diferença quanto à Imax. No entanto, o perfil temporal das amostras é diferente, já que apresentaram variações quanto à permanência do atributo, evidenciadas pelos valores de platô e Td. As curvas tempo-intensidade para as três amostras de água tônica em relação ao gosto amargo são apresentadas na Figura 1.

Tabela 2. Médias dos parâmetros da curva tempo-intensidade para as amostras de água tônica.

\begin{tabular}{|c|c|c|c|}
\hline \multirow[t]{2}{*}{ Parâmetros } & \multicolumn{3}{|c|}{ Amostras } \\
\hline & M-1 & M-2 & M-3 \\
\hline Imax & $5,44^{\mathrm{a}}$ & $5,45^{\mathrm{a}}$ & $6,08^{a}$ \\
\hline Ttot & $74,22^{\mathrm{a}}$ & $72,04^{\mathrm{a}}$ & $67,41^{\mathrm{a}}$ \\
\hline Platô & $9,89^{\mathrm{a}}$ & $12,74^{\mathrm{b}}$ & $13,67^{\mathrm{b}}$ \\
\hline Área & $184,65^{\mathrm{a}}$ & $214,76^{\mathrm{a}}$ & $249,38^{\mathrm{a}}$ \\
\hline TImax & $22,33^{\mathrm{a}}$ & $23,33^{\mathrm{a}}$ & $21,67^{\mathrm{a}}$ \\
\hline $\mathrm{Td}$ & $32,22^{\mathrm{a}}$ & $36,07^{\mathrm{b}}$ & $35,33^{\mathrm{ab}}$ \\
\hline
\end{tabular}

Médias seguidas por pelo menos uma mesma letra, na linha, não diferem entre si ao nível de 5\% de probabilidade pelo teste Duncan.

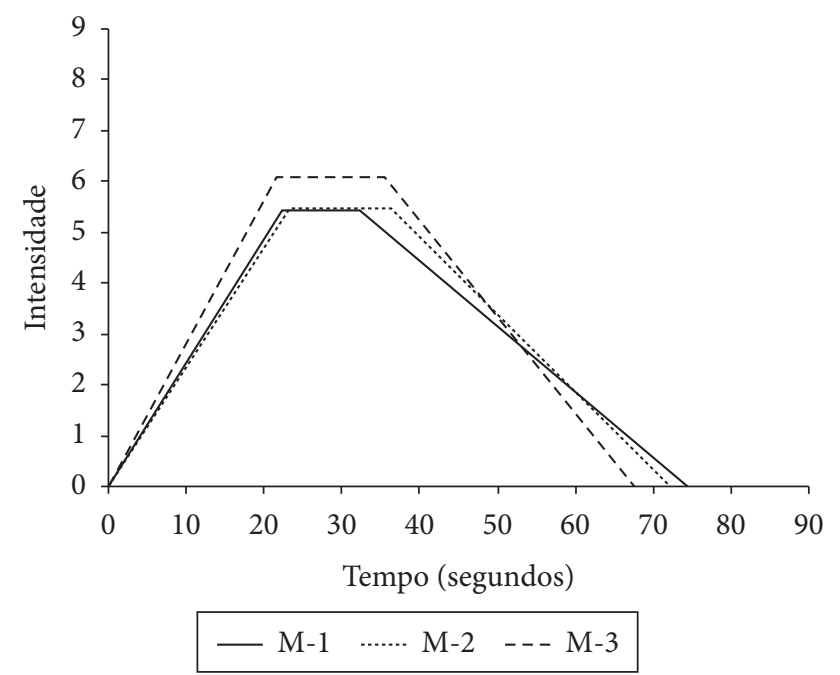

Figura 1. Curva tempo-intensidade característica do gosto amargo para as três amostras de água tônica. 
Tabela 3. Médias dos escores para aceitação das marcas de água tônica.

\begin{tabular}{cc}
\hline Marca & Médias $^{*}$ \\
\hline M-1 & $6,24^{\mathrm{a}}$ \\
M-2 & $6,08^{\mathrm{a}}$ \\
M-3 & $5,14^{\mathrm{b}}$ \\
\hline
\end{tabular}

${ }^{*}$ Médias seguidas por pelo menos uma mesma letra não diferem entre si ao nível de $5 \%$ de probabilidade pelo teste de Duncan.

\subsection{Aceitação}

As médias dos escores para a aceitação das marcas de água tônica comerciais encontram-se expressas na Tabela 3.

As amostras M-1 e M-2 apresentaram médias de aceitação superiores $(\mathrm{p}<0,05)$ à amostra $\mathrm{M}-3$, sugerindo que o perfil temporal não influenciou a aceitação da água tônica, sendo que a maior permanência do gosto amargo não interferiu na aceitação do produto.

Deve-se ressaltar que no teste de aceitação os consumidores não foram instruídos a permanecerem com a amostra na boca por alguns segundos. A aceitação foi realizada somente para o atributo impressão global e, neste caso, outros fatores como, por exemplo, o teor de açúcar e a concentração de gás podem ter sido mais relevantes no julgamento dos consumidores que o gosto amargo.

\section{Conclusões}

As marcas de água tônica avaliadas apresentaram perfis temporais distintos para o gosto amargo. As amostras não apresentaram diferença em relação à intensidade do gosto amargo pela análise tempo-intensidade. Em relação à aceitação, as amostras M-1 e M-2 foram as mais aceitas ( $<<0,05)$.

\section{Referências bibliográficas}

CLIFF, M.; HEYMANN, H. Development and use of time-intensity methodology for sensory evaluation: a review. Food Research International, v. 26, n. 5, p. 375-385, 1993.

CARDEllO, H. M. A. B.; Da SILVA, M. A. A. P.; DAMASIO, M. H. Análise tempo-intensidade dos estímulos doce e amargo de extrato de folha de estévia (stevia rebaudiana (bert.) bertoni) em doçura equivalente a sacarose. Ciência e Tecnologia de Alimentos, v. 19, n. 2, p. 163-169, 1999.

KELLING, S. T.; HALPERN, P. B. Taste flashes: reaction times, intensity and quality. Science, v. 219, p. 412-422, 1983

MINIM, V. P. R. Análise sensorial: estudos com consumidores. Viçosa: UFV, 2006. 225 p.

MONTEIRO, M. A. M. Caracterização da bebida de café (Coffea arabica L.): análise descritiva quantitativa, análise tempointensidade e testes afetivos. Viçosa, 2002. 158 p. Tese (Doutorado em Ciência e Tecnologia de Alimentos) - Universidade Federal de Viçosa - UFV.

REIS, R. C. Iogurte "light" sabor morango: equivalência de doçura, caracterização sensorial e impacto da embalagem na intenção de compra do consumidor. Viçosa, 2007, 143 p. Tese (Doutorado em Ciências e Tecnologia de Alimentos) - Universidade Federal de Viçosa - UFV.

SAS. SAS Software. Version 9.1. Cary, North Carolina: SAS Institute Inc., 1999.

SILVA, A. F. et al. Avaliação do gosto amargo da bebida de café (Coffea arabica L.) orgânico por meio da análise tempo-intensidade. Ciência e Tecnologia de Alimentos, v. 24, n. 3, p. 468-472, 2004.

WIKIPEDIA. Disponível em: <http://pt.wikipedia.org/ wiki/\%C3\%81gua_t\%C3\%B4nica>. Acesso em: 02 julho 2007. 